\title{
Chlamydia screening in general practice: views of professionals on the key elements of a successful programme
}

\author{
Richard Ma, Aileen Clark
}

\begin{abstract}
Objectives Chlamydia trachomatis is a common sexually transmitted infection with serious consequences if not treated. Chlamydia screening pilots in England have established feasibility in primary care but there are currently no examples of good practice in general practice. The objectives of the study were to understand issues of using general practice as a setting for chlamydia screening and to explore ways of implementing a successful screening strategy.

Methods Based on findings of a literature review, a semistructured schedule was constructed to interview a purposive sample of policymakers, consultants in sexual and reproductive health and primary care professionals. A thematic framework was used for qualitative analysis.

Results Twenty-two themes were identified and were ranked in order of word count. The topic that generated most discussion was heterogeneity of knowledge, attitudes and skills in general practice. When broken down by professional group, this topic ranked the highest for practice nurses and consultants in sexual health; general practitioners (GPs) and the chlamydia screening coordinator spoke most about financial incentives while the public health consultant spoke most about access.

Conclusions Most believed screening can and should be done and general practice can offer better population coverage. It needs to have little impact on clinicians' workload, for example, by using urine tests and self-taken vaginal swabs. Financial recognition needs to reflect the administrative costs and the impact on reception staff, but this and the innovative tests might add to the cost of the screening programme. Incentives have to be handled sensitively to reduce inequity among GPs and other services offering screening.
\end{abstract}

J Fam Plann Reprod Health Care 2005; 31(4): 302-306 (Accepted 6 August 2005)

\section{Key message points}

- Key stakeholders confirm chlamydia screening is feasible in general practice.

- General practice offers population coverage that is crucial to the success of the screening programme.

- Self-taken vulval swabs or urine tests would reduce clinicians' involvement and their workload.

- A nationally agreed financial framework to incentivise screening might encourage uptake.

- Nucleic acid amplification tests and financial incentives might add to the cost of the screening programme.

The Village Practice, Islington, London, UK

Richard Ma, MRCGP, MSc, General Practitioner

Public Health and Policy Research Unit, Institute of Community Health Sciences, London, UK

Aileen Clarke, MD, FFPH, Reader

Correspondence to: Dr Richard Ma, The Village Practice, 115 Isledon Road, Islington, London N7 7JJ, UK.

E-mail: richard.ma@btinternet.com

\section{Introduction}

Chlamydia is a common sexually transmitted infection (STI); up to $70 \%$ of women and $50 \%$ of men with genital chlamydia infection are asymptomatic. Screening and treatment for infection have been shown to reduce complications such as pelvic inflammatory disease. ${ }^{1}$ A recent population-based prevalence study in Britain reported that the highest prevalence of Chlamydia trachomatis was among women aged 16-24 years (3.0\%) and men aged 25-34 years $(3.1 \%){ }^{2}$ The UK Health Protection Agency reported that cases of chlamydia rose by $8 \%$ from 2002 to 2003 , with the majority of infections occurring in men and women aged between 20 and 25 years of age. ${ }^{3}$

The National Strategy for Sexual Health and HIV recognised the rising incidences of STIs, HIV and teenage pregnancy and suggested plans to reduce sexual health morbidity; ${ }^{4}$ the implementation plan suggested reduction of chlamydia infection through a national screening programme. ${ }^{5}$ Based on evidence from chlamydia screening pilots in Portsmouth and Wirral, the Department of Health (DH) approved the extension of the screening programme for people under 25 years; this now covers $25 \%$ of all the primary care trusts (PCTs) in England. 6,7 The recent Public Health White Paper entitled 'Choosing Health' 8 has highlighted sexual health as an emerging problem and recommended urgent implementation of chlamydia screening nationally by 2007 .

There are concerns about the ability of general practitioners (GPs) to provide chlamydia screening. ${ }^{9}$ Apart from screening pilot evaluations, there is currently little evidence on screening in UK general practice. ${ }^{10}$ Faced with an emerging public health problem and a lack of guidance on good practice, how should PCTs reconcile the political and public health pressures of extending screening coverage with the concerns of GPs? We undertook a qualitative study to investigate the issues involved and suggest some ways forward.

\section{Methods \\ Sampling}

We used a purposive mixture of criterion and snowball sampling; we chose interviewees to reflect their role from policymaking to experience and/or responsibility for local implementation and delivery of chlamydia screening. Twelve participants agreed to take part: four consultants in sexual and reproductive health [three genitourinary medicine (GUM) and one family planning (FP)], one consultant in public health, a chlamydia screening coordinator, three GPs and three practice nurses (PNs). Of the sample from general practice, three were from practices already commissioned to provide national enhanced service (NES) for sexual health and the remainder have an interest. The chlamydia screening co-ordinator is a member of the National Chlamydia Screening Steering Group (NCSSG) as well as a member of the local steering committee. Two consultants belong to the local steering committee, another is a member of the Independent Advisory Group on Sexual Health and HIV, and another is sexual health lead for the PCT. One consultant who is a member of NCSSG declined to take part due to other commitments. 


\section{Interview schedules}

We used a semi-structured schedule to interview the subjects. The topics on the schedule were drawn from a literature review, which focused on chlamydia screening in general practice. ${ }^{10}$ One of the authors (R.M.) contacted each participant by e-mail, phone or in person and sought consent for face-to-face interviews, which were undertaken between 27 June and 20 August 2004.

\section{Analysis}

The interviews were recorded electronically and transcripts were typed. Respondents were invited to check the accuracy of the transcripts. A variation of thematic analysis was used. ${ }^{11}$ Each transcript was analysed for themes and relevant excerpts were arranged under each theme heading. Subsequent transcripts were searched for materials relating to each theme; new themes that emerged were given new headings and were searched for in previous transcripts. This process was repeated until no new themes emerged. Wherever possible, contradictory views from the participants were sought for each theme heading.

For reporting results, each interviewee was assigned a code for their professional category: CON (for consultant in FP or GUM), GP and PN; to protect anonymity as much as possible, the public health consultant and chlamydia screening co-ordinator were both assigned $\mathrm{PH}$.

\section{Ethical approval}

Ethical approval for the study was granted by the London School of Hygiene and Tropical Medicine ethics committee and Camden and Islington Community Health Services Local Research Ethics Committee.

\section{Results}

In order to view the dataset in context, we produced a bar chart of the word count for each theme (Figure 1). While this is useful to view which themes generated most discussion and debate, we recognise a robust statistical analysis is not possible and advise against interpreting the data quantitatively.

The top three topics that generated most discussion were (in descending rank): variation in knowledge, attitudes and skills in general practice; accessibility and financial incentives. The topic that generated most discussion was different for each professional group: the top ranking theme for GPs and the chlamydia screening coordinator was financial remuneration; for PNs and consultants in sexual health, it was knowledge, attitudes and skills; and for the consultant in public health, accessibility.

\section{Heterogeneity of knowledge, attitudes and skills}

Heterogeneity of knowledge, attitudes and skills was the most discussed topic among PNs and consultants. All three PNs were confident that sexual health skills could be achieved in general practice and they appeared to be more comfortable with raising sexual health issues with patients. One consultant noted GPs have to deal with other sensitive and stigmatising issues such as mental health; skills in dealing with this could be applied to sexual health.

Two of the GPs thought their peers should be able to raise sexual health issues appropriately and know how to take a history but one disagreed.

"Sexual history taking skills are 'learnable' ... can understand how some people find it difficult ... but we can't expect this from all GPs." [GP1]

One solution to increase participation from all GPs was to reduce the need to talk about sex.

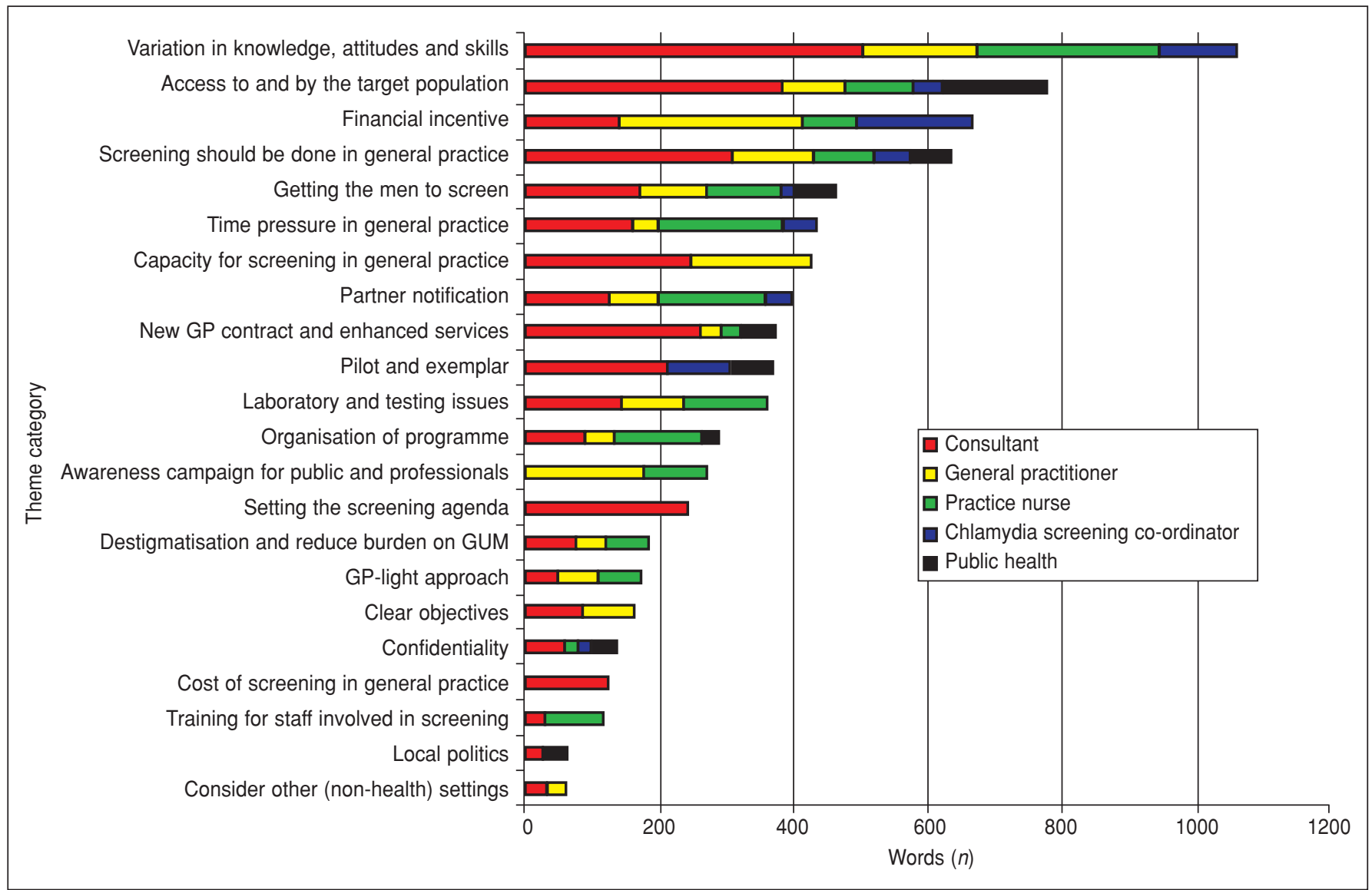

Figure 1 Number of words spoken by each professional by theme 


\section{ARTICLE}

"If we had a 'blanket' screening policy, might make it easier if GPs were not required to discuss full sexual history." [PN2]

\section{Accessibility}

All the consultants thought general practice has access to a greater proportion of the community; only one GP was confident that sexually active people attend general practice; and the public health professionals had opposing views between them.

"NATSAL (National Survey of Sexual Attitudes and Lifestyle - see Fenton et al. ${ }^{2}$ ) shows only $10 \%$ of sexually active adults have visited GUM clinics. General practice is a place that most of the population of all ages can attend." [CON1]

Some consultants thought there wasn't much evidence to support the fact that the sexually active population attend general practice. However, access to a greater proportion of the population does not necessarily mean the same access to the 'at-risk' population

"How do we attract the sex workers and avoid the worried well?" [GP1]

\section{Financial incentives}

While all GPs accepted financial remuneration might need to be discussed, one was very insistent that any extra workload due to screening was recognised, regardless of whom this affects most. Two PNs did not agree and one did not comment. One PN felt chlamydia screening should be part of general personal care, so payment would not be justified. One consultant noted that in the pilot studies the financial incentive might have explained the large number of tests from general practice. The payment to the GPs in the pilot studies was intended primarily to cover data collection costs for research purposes.

"Examples from the pilots in Portsmouth and Wirral showed GPs achieved a high screening coverage ... but they were paid!' [CON2]

One of the public health professionals questioned how remuneration could be justified if the screening test were free and did not have to involve a clinician, neither are GPs participating in the screening programme expected to follow-up results and manage positive cases.

\section{Screening should be done in general practice}

All interviewees agreed there should be a chlamydia screening programme for young people. If this were extended to general practice, the issues appear to be 'how' rather than 'if' this should be done.

\section{Screening men}

Three of the consultants and both public health professionals believed reaching out to young men is a challenge. The consultants and one PN believed more innovative ways must be sought to screen men. One GP needs to be convinced that asymptomatic men need screening. Only one PN specifically mentioned urine sample as a way of screening for men while two of GPs and two PNs thought urethral swabs were used for screening.

\section{Time pressure}

Consultants understood how GPs feel overwhelmed with working under the new contract and other initiatives such as National Service Frameworks. One was wary there are no studies looking at how chlamydia screening affected GPs.
PNs argued sexual health is an essential component of health promotion and should not be ignored. One GP felt if screening only involved doing a quick swab or urine sample then the potential for opportunistic health promotion might be missed. One public health professional stated as most of the information is enclosed in the screening kit, the clinician would not have to spend time talking about the test.

\section{Capacity}

The experience of the pilots highlighted impact on receptionists who identified screening subjects upon their arrival. It might be different in the case of opportunistic screening where the clinician might suggest screening. The consultants could not agree on the likely impact on reception or clinical staff while two of the GPs anticipated increased workload for both; the PNs did not comment.

\section{Partner notification}

Although some of them have performed this before, GPs and PNs were concerned about the volume of partner notification from the screening. The chlamydia screening office has been co-ordinating the treatment and partner notification for positive cases in the local screening programme so this should not be an issue for most GPs.

New GP contract, enhanced services and equity

Expressions of interest to provide enhanced services in sexual health were received by over half of the practices in Islington (Islington PCT locality director, personal communication). This opportunity was recognised by three consultants, two GPs, one PN and a public health professional, but they were cautious with this development. While this might facilitate more diagnoses and treatment of STIs in the community, it could be misinterpreted as a 'screening' service and cause confusion and divisiveness between those practices being paid for this service and others who are not. Two consultants noted that practices that are doing some STI testing might stop completely if they were not financially recognised.

"The [NES] ... might attract innovative practices. There are perverse effects - those who already do some work might do less (if not commissioned)." [CON4]

A GP raised the issue of equity of access for patients who are registered with a non-NES practice; this was echoed by a public health professional who was also concerned with commissioning enhanced service from all GPs if quality is going to be poor in some.

\section{Pilot and exemplar}

Two consultants commented evaluations from the screening pilots and local screening programmes would help PCTs decide on a strategy for general practice. One suggested screening should start in a small number of practices, as this would hopefully put 'peer pressure' on the rest to conform. Another suggested piloting it in practices already providing sexual health NES and rolling it out after evaluation.

\section{Laboratory and tests}

GPs, PNs and consultants agreed that the ease of administering a test should facilitate the screening programme; urine tests for men and self-taken vulvalvaginal swabs for women might increase acceptance for screening and would lessen impact on clinicians' workload. However, as these tests are more expensive and technically difficult, not all of the local laboratories can process them. 


\section{Programme organisation}

Although current screening programme for chlamydia suggests an 'opportunistic' approach, PNs, GPs and consultants have different views on how the screening programme should be organised.

"Should have a population-based approach, similar to cytology - centrally based so little impact on admin staff." [PN1]

"Unsure how 'opportunistic'screening is defined-perhaps at the discretion of the clinician." [PH1]

"Blanket approach is not appropriate ... must let people manage their own health ... would prefer a model where people are able to ask for a test and not have it imposed on them. Opportunistic screening is not feasible in general practice." [GP1]

\section{Information campaign}

One GP was keen for individuals to take responsibility to screen, and would like to see public campaigns and invitation letters sent out to patients to increase awareness. This would help some clinicians by reducing the need to discuss issues they felt uncomfortable about.

\section{Advocates for screening agenda}

Two consultants were keen for advocates in sexual health to put pressure on PCTs to consider and fund screening in general practice and other venues. They recognised that PCTs have competing priorities and encouraged practitioners to help agenda setting.

"Sexual health practitioners need to present coherent arguments involving rationale, costing and examples of good practice. We need to sell this problem in a 'package'." [CON1]

\section{De-stigmatising STI and reducing burden on GUM}

The consultants and PNs did not think GUM clinics had the capacity to offer population-wide screening for STIs. They agreed general practice could help reduce the burden by offering screening to asymptomatic populations.

"It is not helpful to propagate the idea of 'special infections' that need to be treated in a 'special place' ... we need to demystify STIs among GPs, secondary care colleagues and the public." [CON3]

One consultant and a PN suggested that screening outside GUM, especially in non-health settings, might help de-stigmatise and demystify STIs in the community.

"Debatable if STI testing in general practice will destigmatise it ... how about screening at pharmacists?" [GP1]

\section{'GP-light' approach}

Clinicians might need training to be confident with raising STI screening opportunistically, but it might not be an effective use of a GP's time to do so.

"Obtaining better skills would take time and effort, so a screening strategy that does not involve too much of GPs' time might help facilitate this - something that is 'GPlight'”. [CON4]

\section{Clear objectives}

One consultant and a GP were unclear about the objectives of the screening programme. The consultant questioned if this was a step to solve access to sexual health services and one GP was worried this would give out out wrong message on testing for other STIs.

\section{Confidentiality}

Although raised by public health, a consultant and a PN, confidentiality with respect to a screening programme was not seen as a major issue, but recognised that STI testing in a GUM setting is more anonymous.

\section{Costing of screening programme}

Two consultants speculated that the cost of screening might increase if rolled out into general practice and would value an economic analysis of this approach.

Training

All PNs and a GP were interested in multidisciplinary training events on topics such as: confidentiality, under-16s and wider sexual health issues. Such events have been organised by the PCT on different topics and they would value one on sexual health.

Politics

Public health and a consultant recognised attitudes and working cultures between GUM and GPs might potentially make sexual health work in primary care a difficult issue.

\section{Other venues}

One consultant and a GP mentioned other venues for screening must be explored and that pharmacist and nonhealth venues are possible options. The DH is now running a pilot in high street pharmacies in London and Cornwall. ${ }^{12}$

\section{Limitations of the study}

Interviews in qualitative research allow more in-depth discussion of the research topic but generalisability is difficult due to the uniqueness of the subjects and contextual factors. We believe other GPs and sexual health professionals are likely to share concerns expressed in this study but we accept this may only be applicable to urban areas.

We acknowledge sampling in qualitative research will not give a representative view of the study population. It might have been useful to involve GPs and PNs with little experience or interest in sexual health and consultants who did not feel chlamydia screening should be offered in general practice.

\section{Conclusions}

The interviews from this study strongly suggest that chlamydia screening is feasible in general practice. Results from the first year of screening showed $10 \%$ of positive cases were from general practice and 63\% from family planning clinics but the positivity rates were the same $(10 \%){ }^{13}$ The chlamydia screening pilots showed most of the infections were from general practice (ranging from $25 \%$ to $50 \%){ }^{7}$ Together with evidence that sexually active population are attending general practice, ${ }^{14,15}$ it is therefore crucial to involve general practice for a good coverage.

It appears a screening programme needs to be organised in a way that demands little time and training from clinicians to increase coverage and maintain quality. Tests using urine samples and self-taken vulval-vaginal swabs may encourage uptake of screening and reduce the need for clinician input. From economic modelling, reducing clinician input has been shown to reduce the cost of screening. 16 The screening pilots in England showed that reception staff were central to the opportunistic screening strategy so their support would be crucial. ${ }^{17}$

Financial incentives have been shown to facilitate public health interventions in general practice such as immunisations and cervical cytology. There is currently no 


\section{ARTICLE/NEWS ROUNDUP}

guidance from the $\mathrm{DH}$ on remuneration for GPs for chlamydia screening. There needs to be a nationally agreed pricing framework for chlamydia screening in general practice to encourage uptake and reduce inequity and resentment among GPs and other services which also provide screening.

However, use of innovative tests and financial incentives would almost certainly add cost to the screening programme; we therefore recommend an economic analysis to evaluate these strategies.

\section{Acknowledgements}

This work was undertaken with the support of Islington PCT, which received funding from the NHS Executive. The views expressed in this publication are those of the authors and not necessarily those of the NHS Executive.

This paper is based on a thesis submitted to the London School of Hygiene and Tropical Medicine for the degree of MSc in Public Health

This paper was presented at the 8th GUM Non-Consultant Career Grade (NCCG) Conference held at Loughborough University, Loughborough, UK in September 2005.

The authors would like to thank the interviewees for giving up their time to participate in this research, and to Dr Chris Wilkinson for commenting on the final draft.

\section{Statements on funding and competing interests}

Funding. None identified.

Competing interests. Richard Ma works as a GP in Islington and is a member of Islington PCT Sexual Health Steering Group.

References

1 Scholes D, Stergachis A, Heidrich FE, Andrilla H, Holmes KK, Stamm WE. Prevention of pelvic inflammatory disease by screening for cervical chlamydial infection. N Engl J Med 1996; 334: 1362-1366.

2 Fenton KA, Korovessis C, Johnson A, McCadden A, McManus S, Wellings K, et al. Sexual behaviour in Britain: reported sexually transmitted infections and prevalent genital Chlamydia trachomatis infection. Lancet 2001; 358(9296): 1851-1854.

3 Health Protection Agency. HIV and Other Sexually Transmitted Infections in the United Kingdom in 2003 Annual Report. London, UK: Health Protection Agency, November 2004. http://www.hpa.org.uk/infections/topics_az/hiv_and_sti/publications/ annual2004/annual2004.htm [Accessed 18 February 2005].

4 Department of Health. National Strategy for Sexual Health and HIV.
London, UK: HMSO, 2001.

5 Department of Health. The National Strategy for Sexual Health and HIV: Implementation Action Plan. London, UK: HMSO, 2002. http://www.dh.gov.uk/assetRoot/04/06/55/43/04065543.pdf [Accessed 18 February 2005].

6 Pimenta JM, Catchpole M, Rogers PA, Perkins E, Jackson N, Carlisle $\mathrm{C}$, et al. Opportunistic screening for genital chlamydial infection. I: Acceptability of urine testing in primary and secondary healthcare settings. Sex Transm Infect 2003; 79: 16-21.

7 Pimenta JM, Catchpole M, Rogers PA, Hopwood J, Randall S, Mallinson $\mathrm{H}$, et al. Opportunistic screening for genital chlamydial infection. II: Prevalence among healthcare attenders, outcome, and evaluation of positive cases. Sex Transm Infect 2003; 79: 22-27.

8 Department of Health. Choosing Health: Making Healthier Choices Easier (Public Health White Paper). London, UK: Department of Health, November 2004

9 McNulty C, Freeman E, Bowen J, Shefras J, Fenton K. Barriers to opportunistic chlamydia testing in primary care. Br J Gen Pract 2004; 54: $508-514$.

10 Ma R. How should Islington Primary Care Trust implement screening for genital Chlamydia trachomatis in general practice? MSc dissertation, London School of Hygiene and Tropical Medicine, London, UK, 2004 [available at LSHTM library; shelf mark MSC 4427].

11 Forrest Keenan K, van Teijlingen E, Pitchforth E. The analysis of qualitative research data in family planning and reproductive health care. J Fam Plann Reprod Health Care 2005; 31: 40-43.

12 Department of Health press release. 'Chlamydia screening on the high street'. Department of Health, London, UK, 8 February 2005.

13 LaMontagne DS, Fenton KA, Randall S, Anderson S, Carter P on behalf of the National Chlamydia Screening Steering Group. Establishing the national chlamydia screening programme in England: results from the first full year of screening. Sex Transm Infect 2004; 80: 335-341.

14 Cassell JA, Brook MG, Mercer CH, Murphy S, Johnson AM. Treating sexually transmitted infections in primary care: a missed opportunity? Sex Transm Infect 2003; 79: 134-136.

15 Dawe F, Rainford L. Contraception and Sexual Health 2003. London, UK: Office for National Statistics, 2004. http://www.dh.gov.uk/ assetRoot/04/08/99/46/04089946.pdf [Accessed 15 February 2005].

16 Adams EJ, LaMontagne DS, Johnston AR, Pimenta JM, Fenton KA, Edmunds WJ. Modelling the healthcare costs of an opportunistic chlamydia screening programme. Sex Transm Infect 2004; 80: 363-370.

17 Perkins E, Carlisle C, Jackson N. Opportunistic screening for Chlamydia in general practice: the experience of health professionals. Health Soc Care Community 2003; 11: 314-320.

\section{News Roundup}

\section{US abortion law}

A new law, previously blocked in 1999 , came into effect in the state of Florida on 30 June 2005 Doctors in Florida now by law must notify parents by phone, in person or by certified mail before performing an abortion on a minor (under 18 years of age). They can, however, continue with abortion if it is a medical emergency and judges can also grant waivers in certain circumstances

Reported by Laura Patterson, MRCGP, DFFP GP, Cirencester, $U K$

\section{Cervical screening in Ireland}

Cervical cancer death rates in the Irish Republic have been slowly increasing since 1950 and are now around 73 deaths per year. At present, cervical screening is only provided on an opportunistic basis. Two recent reports were discussed at the first Irish national screening conference held on 18 June 2005 at the University of Limerick. The reports were written by Dr Euphemia McGoogan, an international expert on cervical screening from Edinburgh, and Geraldine Luddy, Director of the Women's Health Council in Ireland. Both reports recommend an extension of the MidWestern Health Board pilot project, which provides 5-yearly screening for women aged 25-60 years. The Department for Health in the Irish Republic is fully committed to rolling out a national programme but as yet is unable to say when screening will be provided nationally.

Source: http://www.icsp.i

Reported by Laura Patterson, MRCGP, DFFP $G P$, Cirencester, $U K$

\section{Abortion time limit}

Members of the British Medical Association, at their recent conference, have voted against a proposal to reduce the time limit for abortion from 24 to 20 weeks' gestation. Less than $1 \%$ of terminations are carried out at or over 22 weeks' gestation.

The last time we saw a reduction in the legal limit, from 28 to 24 weeks' gestation, was in 1990. Improvements in medical care do mean that infants born around 24 weeks' gestation have a much better chance of survival now than ever before. Those who oppose a reduction in the legal limit fear that women would be forced to seek abortion abroad or carry unwanted pregnancies.

Source: http://www.bma.org.uk/ap.nsf/content/abortion.

Reported by Laura Patterson, MRCGP, DFFP GP, Cirencester, UK

\section{Steroids may reduce the risk of miscarriage}

A team of researchers in Liverpool tested whether prednisolone could prevent miscarriage by reducing the number of natural killer (NK) cells in the endometrium. Women with a history of recurrent miscarriage were investigated with endometrial biopsy. If the endometrium had high levels of NK cells, the women were offered oral prednisolone during the first 21 days of the menstrual cycle. A second endometrial biopsy on Day 21 showed a reduction in the number of NK cells. Of the 29 women who took part, seven became pregnant and two have delivered healthy babies

The treatment is promising but there is concern that certain private fertility clinics are offering 'NK kits' to vulnerable women when it is an unproven technique. Dr Quenby, who is leading the research, commented: "There are many unanswered questions at present, and we hope that randomised controlled trials will shed more light on the mechanisms involved and whether the use of prednisolone may, in fact, represent a new and effective treatment for recurrent miscarriages".

Source: http://pregnancyandbaby.com/read/articles/5546.htm.

Reported by Henrietta Hughes, MRCGP, DFFP GP, London, UK

\section{Upward trend in STIs}

The latest figures from the Health Protection Agency (HPA) show that sexually transmitted infections (STIs) are continuing to rise. There was an overall increase of $2 \%$ in 2004 compared with the previous year. The HPA said the figures were 'disappointing', but that the rate of rise had slowed and some infections were less prevalent. Chlamydia rose by $8 \%$ and syphilis by $37 \%$. New cases of gonorrhoea fell by $10 \%$.

Source: http://www.hpa.org.uk.

Reported by Henrietta Hughes, MRCGP, DFFP GP, London, UK 